\title{
酒精性肝病合并药物性肝损伤的代谢组表征 和生物标志物研究
}

王肖辉 ${ }^{1,2 \dagger}$ ，景婧 ${ }^{2 \dagger}$ ，牛明 ${ }^{2}$ ，卫璐戈 ${ }^{2}$, 刘晓熠 ${ }^{2}$, 涂灿 $^{2}$, 柏兆方 ${ }^{2}$, 周元园 ${ }^{2}, 弓^{2}$ 乐 $^{2}$, 朱敬肖 ${ }^{2}$, 肖小河 ${ }^{4 *}$, 邹正升 ${ }^{3 *}$, 王伽伯 ${ }^{2 *}$

1. 湖南中医药大学药学院, 长沙 410200 ;

2. 解放军302医院全军中医药研究所, 北京 100039 ;

3. 解放军302医院非感染性肝病诊疗与研究中心, 北京 100039 ;

4. 解放军302医院中西医结合诊疗与研究中心, 北京 100039

$\dagger$ 同等贡献

*联系人: E-mail: pharmacy302@126.com; zszou302@163.com; wjb0128@126.com

收稿日期：2019-03-11; 接受日期：2019-04-03; 网络版发表日期：2019-05-20

国家重大新药创制专项课题(批准号：2015ZX09501-004-001-008)、国家自然科学基金(批准号：81503247)、北京市科技新星计划项目(批准 号: Z16111000490000)、国家公益性行业专项课题(批准号: 201507004-04)和国家中药标准化项目(批准号: ZYBZH-Y-BJ-07)资助

摘要随着社会经济的发展, 在酒精性肝病(alcoholic liver disease, ALD)基础上发生药物性肝损伤(drug-induced liver injury, DILI)的问题日渐突出，然而ALD合并DILI(ALD-DILI)究竟是何者起主导作用时常难以界定，其损伤 通路机制亦不完全清楚。本文对临床血清标本进行代谢组学表征发现, ALD-DILI在代谢组水平上与DILI整体类 似(差异化合物60个)，而与ALD相差较大(差异化合物1232个); 对差异代谢物进行鉴定及代谢通路分析发现，相较 于单纯的DILI, ALD-DILI患者体内胆汁酸、氨基酸、脂质合成分解等功能性损伤更严重，与本课题组前期观察 的临床表型一致。进一步篮选到可用于区分DILI与ALD-DILI的4个候选生物标志物(Phosphatidate, Phosphatidylethanolamine, Taurolithocholate, 3-Ketolactose), ROC曲线下面积均大于 0.8 ; 其中Phosphatidate分别与 3-Ketolactose, taurolithocholate的峰面积比值具有更好的区分诊断效果, ROC曲线下面积分别达 0.918 和 0.886 , 且比值法有 利于降低样本处理与检测仪器等系统误差, 能较好地辅助临床早期鉴别诊断有酒精性肝病的药物性肝损伤, 并指 导临床合理用药.

关键词药物性肝损伤, 酒精性肝病, 代谢组学, 生物标志物, 诊断

随着人们生活的改善，我国饮酒者乃至嗜酒者逐 年增多, 由酒精所致肝损伤的发病率呈逐渐上升的趋 势 ${ }^{[1,2]}$. 最新流行病调查发现, 酒精性肝病(alcoholic li- ver disease, ALD)在全世界范围内也成为严重危害公 众健康的常见疾病之一, 并呈逐年增加趋势 ${ }^{[3 \sim 5]}$. 另外, 药物性肝损伤(drug-induced liver injury, DILI)为临床

引用格式: 王肖辉, 景婧, 牛明, 等. 酒精性肝病合并药物性肝损伤的代谢组表征和生物标志物研究. 中国科学: 生命科学, 2019, 49: 749-760 Wang X H, Jing J, Niu M, et al. Metabolomic profiling and biomarkers of drug-induced liver injury with pre-existing alcoholic liver disease (in Chinese). Sci Sin Vitae, 2019, 49: 749-760, doi: 10.1360/N052018-00291 
常见的严重药物不良反应之一，而在酒精的影响下可 能会使肝脏对药物毒性更加敏感 ${ }^{[6]}$. 例如, 一份病例报 告显示，长期饮酒可增强低剂量对乙酰氨基酚的肝毒 性 $^{[7]}$. 本课题组前期研究发现, 在有慢性基础肝病的药 物性肝损伤患者中，酒精性肝病是慢性基础肝病的主 要类型, 并且ALD-DILI的治愈率较单纯的DILI低. 根 据美国药物诱导肝损伤网络(Drug Induced Liver Injury Network, DILIN)公布的数据, 有慢性基础肝病的DILI 患者总死亡率 $(19.0 \%)$ 高于无慢性基础肝病的患者 $(8.1 \%)^{[8]}$. 临床上对于ALD合并DILI患者的诊断、治 疗与转归等难以评估, 对于其发生与发展究竟是谁起 主导作用难以界定. 如何评价与诊断ALD-DILI, 指导 临床用药与管理是临床上迫切需要解决的问题. 然而, 临床上对DILI的诊断十分困难, 特别是早期鉴别ALDDILI与某些有长期饮酒史但没有造成ALD的DILI患 者之间的诊断. 目前对于DILI与ALD-DILI之间的差异 机制研究并不完全清楚. 因此，阐明ALD-DILI与DILI 的生物组学水平上的差异, 并寻找可辅助鉴别诊断的 标志物具有现实意义.

本课题组 ${ }^{[9]}$ 前期以单中心病例为基础，采用回顾 性研究发现, 在临床生化指标表型上, ALD-DILI更接 近于单纯的DILI, 而与ALD相差较大. 但是这三种疾病 在生物组学水平上的差异尚不清楚, 限制了理解ALDDILI疾病的生物学机制以及临床鉴别诊断. 因此, 本文 在此基础上，通过收集ALD合并DILI、单纯的DILI和 匹配的ALD患者血清标本，采用代谢组学的方法系统 表征了DILI，ALD-DILI，ALD的代谢组特征谱差异， 篮查DILI与ALD-DILI的生物标志物，分析ALD-DILI 与DILI之间的代谢差异，以期在临床上可辅助早期鉴 别诊断ALD-DILI与DILI, 并指导临床合理用药.

\section{1 材料与方法}

\section{1 一般资料}

病例样本来源：检索中国人民解放军第 302 医院 2015年6月 2017年6月非感染科患者病例数据库，满 足研究的纳入/排除标准, 详细记录受试者性别、年 龄、既往史等信息，并进行生化和肝炎系列检测．采 用回顾性分析进行统计篮选，共90例留有血清样本的 病例纳入本研究，其中酒精性肝病合并药物性肝病患 者血清10例，药物性肝病患者血清40例，酒精性肝病
患者血清40例, 具体篮选流程见图1所示. 样品采集前 未行特异性治疗. 所有研究对象皆签署知情同意书, 研 究方案通过医院伦理委员会审批.

(1) 纳入标准. ( i ) DILI纳入标准: 参照中华医学 会《药物性肝损伤诊治指南》 ${ }^{[10]}$ ，有关中药肝损伤诊 断参照国家药品监督管理局《中药药源性肝损伤临床 评价技术指导原则》 ${ }^{[11,12]}$ 及中华中医药学会《中草药 相关肝损伤临床诊疗指南》 ${ }^{[13,14]}$, 确诊及样品采集前 为经特异性治疗，签署知情同意书，自愿参加本项研 究. (ii) ALD纳入标准: 参照中华医学会《酒精性肝 病诊疗指南》 ${ }^{[15]}$, 确诊及样品采集前为经特异性治疗, 签署知情同意书，自愿参加本项研究. (iii) ALD-DILI 纳入标准: 参照DILI诊断标准被确诊为DILI, 同时参照 ALD诊断标准患有ALD的患者，确诊及样品采集前为 经特异性治疗, 签署知情同意书, 自愿参加本项研究.

（2）排除标准. 患者符合以下任何一条即予排除. ( i ) DILI合并ALD外的其他疾病, 如合并病毒感染性 疾病、非酒精性脂肪性肝病等疾病者; (ii) 合并心血 管、肝、肾、消化、造血系统等严重性疾病者; (iii) 进展性恶性肿瘤或其他严重消耗性疾病，易合并感染 及出血者; (iv) 意识不清, 不能表达主观不适症状, 以 及精神病患者; (v) 孕妇及哺乳期妇女; (vi) 抽烟、吸 毒及嗜酒者.

(3) 伦理学审查. (i ) 研究方案由研究人员共同商 定, 报伦理委员会审批后实施. (ii) 按照《赫尔辛基宣 言》并参照《药品临床研究管理规范》的要求, 制订 知情同意书. 向志愿者提供研究的详细情况, 内容包 括研究目的、方法及过程. 向志愿者明确说明其个人 资料均属保密，若发生与研究有关的损害，志愿者可 得到适当的补偿. 在得到志愿者或其法定代表监护人 的签名并注明日期后方可进行研究. 若志愿者或其法 定代表监护人均无阅读能力, 则应有一名见证人在场, 经详细解释知情同意书后，志愿者或其法定代表监护 人口头同意后, 由见证人签名并注明日期.

\section{2 仪器与试剂}

Agilent Technology 1290 Infinity HPLC高效液相 色谱仪(美国); Agilent Technology iFunnel 6550 Q-TOF LC/MS 四级杆串联飞行时间质谱; 1290 二极光阵列检 测器(G4212A); Agilent Masshunter工作站; 冷冻离心机 (Sigma, 德国); 真空干燥器; 内祄管; 浴旋混匀器(HYQ 


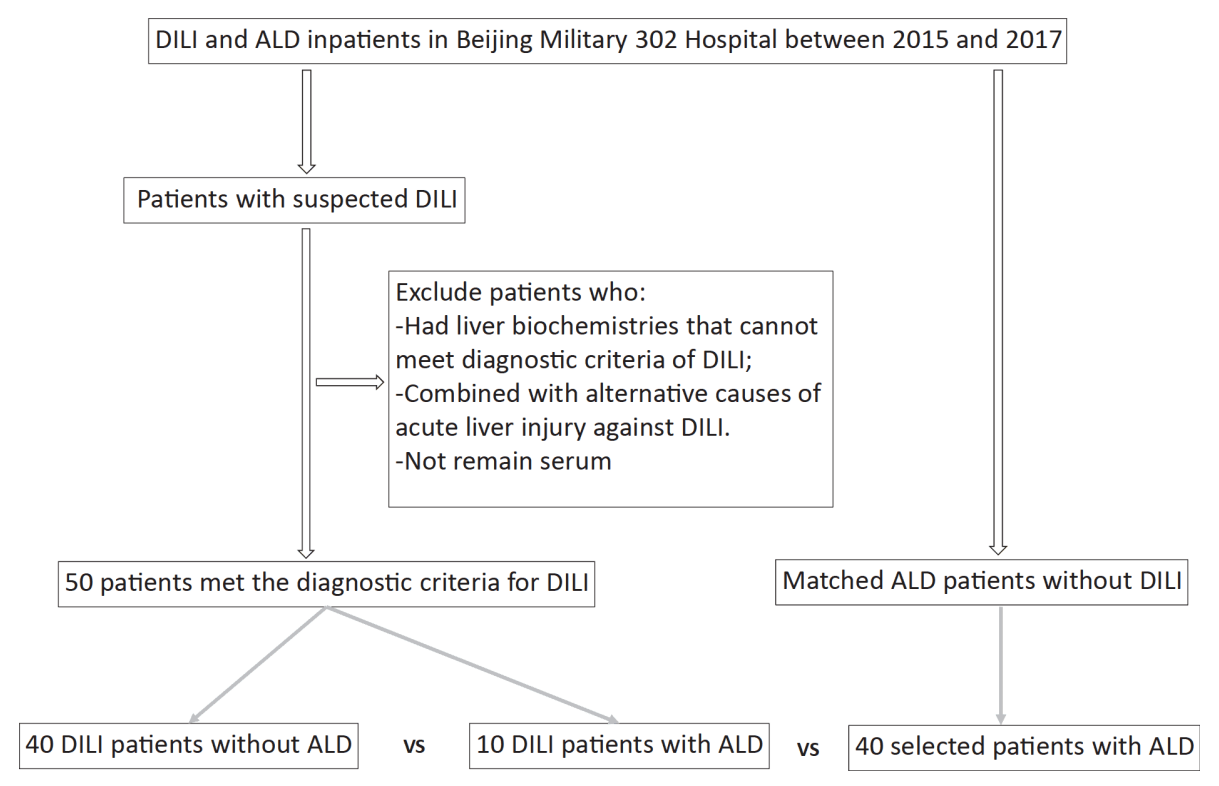

图 1 90例样本纳入流程图. ALD, 酒精性肝病; DILI, 药物性肝损伤

Figure 1 Flowchart depicting the process for cases enrollment. ALD, alcoholic liver disease; DILI, drug-induced liver injury

2121A); 甲醇(色谱级，德国Merck公司); 纯净水(娃哈 哈集团有限公司).

(1) 色谱条件. 色谱柱: ZORBOX RRHD C18分析 柱( $2.1 \mathrm{~mm}$ i.d. $\times 100 \mathrm{~mm}, 1.8 \mu \mathrm{m}$ i.d., Agilent Technologies, 美国). 柱温: $30^{\circ} \mathrm{C}$. 样品温度: $4^{\circ} \mathrm{C}$. 流动相 $\mathrm{A}$ : 水 (加有 $0.1 \%$ 甲酸), 流动相 $\mathrm{B}$ : 乙腈 (加有 $0.1 \%$ 甲酸). 流 速: $0.30 \mathrm{~mL} / \mathrm{min}$; 进样量: $4 \mu \mathrm{L}$. 色谱梯度洗脱条件: $0 \sim 1 \mathrm{~min}, 100 \%$ (A); 1 9 min, 100\% 60\% (A); 9 19 $\mathrm{min}, 60 \% \sim 10 \%$ (A); 19 21 $\mathrm{min}, 10 \% \sim 0 \%$ (A); 21 25 min, $100 \%$ (B).

(2) 质谱条件. 采用具有正和负离子模式的电喷雾 电离源(ESI)的Agilent 6550 Q-TOF LC/MS, 采集模式: ESI Continuum模式. 电喷雾源参数固定如下: 电喷雾 毛细管电压在负电离模式下为 $3.5 \mathrm{kV}$, 在正电离模式 下为 $4 \mathrm{kV}$. 质量范围设定为 $m / z \quad 80 \sim 1000$. 正与负电离 模式下气体温度均为 $225^{\circ} \mathrm{C}$. 气流为 $13 \mathrm{~L} / \mathrm{min}$. 雾化器 设定为 $20 \operatorname{pisg}$ (阴性) 和 20 pisg(阳性)。鞘气温度为 $275^{\circ} \mathrm{C}$, 鞘气流量为 $12 \mathrm{~L} / \mathrm{min}$. 在负模式和正模式下, 喷 嘴电压均为 $2000 \mathrm{~V}$. 质量数校正: 正模式下参考质量 121.0509(Purine, $\left[\mathrm{C}_{5} \mathrm{H}_{4} \mathrm{~N}_{4}+\mathrm{H}\right]^{+}$)和922.0098(HP-0921, $\left[\mathrm{C}_{18} \mathrm{H}_{18} \mathrm{O}_{6} \mathrm{~N}_{3} \mathrm{P}_{3} \mathrm{~F}_{24}+\mathrm{H}\right]^{+}$), 负模式下使用 112.9856 $\left(\mathrm{TFANH}_{4}, \quad\left[\mathrm{C}_{2} \mathrm{H}_{4} \mathrm{O}_{2} \mathrm{NF}_{3}-\mathrm{NH}_{4}\right]^{-}\right)$和1033.9881 TFANH4 $+\mathrm{HP}-0921,\left[\mathrm{C}_{20} \mathrm{H}_{22} \mathrm{O}_{8} \mathrm{~N}_{4} \mathrm{P}_{3} \mathrm{~F}_{27}-\mathrm{NH}_{4}\right]^{-}$).

\section{3 临床标本的处理}

将存放于 $-80^{\circ} \mathrm{C}$ 冰箱中DILI血清样品, $4^{\circ} \mathrm{C}$ 复融, 精密吸取 $200 \mu \mathrm{L}$ 置于EP管中, 加入3 倍量的预冷的甲 醇稀释, 浴旋混匀, 在 $4^{\circ} \mathrm{C}$ 下以 $12000 \mathrm{r} / \mathrm{min}$ 离心 $10 \mathrm{~min}$, 过 $0.22 \mu \mathrm{m}$ 微孔滤膜, 即为备用样品; 质控样品 (quality control, QC) 是从每个待分析的样本取 $10 \mu \mathrm{L}$ 混匀制备 所得.

\section{4 数据处理统计学分析}

所有数据均由安捷伦Masshunter Profinder软件进 行预处理后导入MetaboAlalyst 3.0在线分析网站进行 进一步数据过滤和归一化处理. 将归一化得到数据导 入SIMCA-P 14.0软件进行主成分分析(principal component analysis, PCA)和正交校正偏最小二乘法-判别 分析(partial least squares discrimination analysis, OPLSDA). PCA作为一种无监督的模式识别方法, 通过将分 散在变量中的信息集中到主成分上来描述数据内部结 构, 得到PCA得分图直观显示不同组别生物体代谢模 式的变化，具有相同或相似状态的样本处于相近的位 置. 结合ANOVA及倍数变化分析 $(P<0.05$ 且 Fold change $(\mathrm{FC})>1.5$ 或 $<0.5)$, 篎选出具有显著性差异的代 谢物(VIP $>1$ 和 $\mid P($ corr $) \mid \geq 0.5)$. 将正负离子篮选出的差 
异代谢物在Human Metabolome Database和Mass Hunter PCDL Manager(MHPM)等生物学数据库进行比对 篮查鉴定和代谢通路分析.

采用IBM SPSS Statistics 22.0进行统计分析, 符合 正态分布的计量资料用 $x \pm s$ 表示, 组间比较采用独立样 本 $T$ 检验; 不符合正态分布的计量资料用 $M(Q 1, Q 3)$ 表 示, 组间比较采用非参数检验.

\section{2 结果}

\section{1 临床特征分析}

病例分析(见表1)显示: 从性别上来看, ALD-DILI 患者与DILI患者差异较大 $(P<0.001)$, 与ALD患者相比 无差异(均是男性占比较高). 这可能与男性比女性饮 酒乃至嗜酒居多有关. 而年龄上三组之间并无显著性 差异 $(P=0.549)$. 三组肝脏生化指标(包括ALT, AST, ALP, TB, GGT, TC, TG, INR，血白蛋白和胆碱酯酶)
比较结果显示, 整体上ALD-DILI的患者与DILI患者更 类似; 其中某些指标, 如ALP, TB, GGT, INR等指标, 均 较DILI, ALD患者更为严重 $(P$ 均 $<0.05)$.

\section{2 代谢组学的多元统计分析}

分析ALD-DILI, DILI, ALD之间的差异化合物. 采 用代谢组学分析方法，对正、负离子模式下的患者体 内代谢化合物进行PCA和OPLS-DA识别分析. PCA分 析(图2A, B)所示, 可观察到在正离子模式下, $\mathrm{QC}$ 组较 好地聚集在得分矩阵投影图中间附近位置, 表明在分 析过程中LC-MS系统稳定. 另外, 可观察到ALD-DILI 与ALD, DILI与ALD之间有比较好的区分度, 表明 ALD-DILI, DILI均与ALD有显著性代谢差异. 从代谢 组学水平初步验证了课题组前期研究的结果, 即ALDDILI与DILI更类似. 为了进一步区分ALD-DILI与DILI 间代谢物的差异, 通过使用有监督的模式识别方法 OPLS-DA进行分析, 结合VIP $>1$ 和 $\mid P($ corr $) \mid \geq 0.5$ 篮选,

表 1 DILI, ALD, ALD-DILI患者的临床生化指标*

Table 1 The characteristics of DILI patients compared to those of DILI patients with pre-existing ALD and matched ALD patients

\begin{tabular}{|c|c|c|c|c|c|c|c|}
\hline 指标 & DILI组 $(n=40)$ & ALD-DILI组 $(n=10)$ & ALD组 $(n=40)$ & $P$ value $\mathrm{a}^{\text {a) }}$ & $P$ value $\mathrm{e}^{\mathrm{b})}$ & $P$ value ${ }^{\mathrm{c})}$ & $P$ value ${ }^{\mathrm{d})}$ \\
\hline 性别 (\%) & $19(47.5 \%)$ & $8(80 \%)$ & $35(87.5 \%)$ & $<0.001$ & $<0.001$ & $<0.001$ & 1.00 \\
\hline 年龄 (年, 中位数[IQR]) & $43.50(29.50,57.00)$ & $43.50(34.25,52.00)$ & $49.00(42.25,54.00)$ & 0.549 & - & - & - \\
\hline $\begin{array}{c}\text { 丙氨酸转氨酶 }\left(\mathrm{U} \mathrm{L}^{-1}, \text { 中位数 }\right. \\
[\mathrm{IQR}])\end{array}$ & $\begin{array}{l}1240.00(840.5 \\
1453.73)\end{array}$ & $\begin{array}{l}1238.00(769.00 \\
1484.50)\end{array}$ & $31.00(20.00,133.75)$ & $<0.001$ & 1.00 & 0.002 & $<0.001$ \\
\hline $\begin{array}{c}\text { 天冬氨酸转氨酶 }\left(\mathrm{U} \mathrm{L}^{-1} \text {, 中位数 }\right. \\
[\mathrm{IQR}])\end{array}$ & $\begin{array}{l}829.00(506.50 \\
1048.28)\end{array}$ & $\begin{array}{l}716.50(456.75 \\
1331.50)\end{array}$ & $49.00(31.00,191.00)$ & $<0.001$ & 1.00 & $<0.001$ & 0.001 \\
\hline $\begin{array}{c}\text { 碱性磷酸酶 }\left(\mathrm{U} \mathrm{L}^{-1} \text {, 中位数 }\right. \\
[\mathrm{IQR}])\end{array}$ & $\begin{array}{l}213.00(151.50 \\
275.00)\end{array}$ & $\begin{array}{l}255.50(177.40 \\
331.00)\end{array}$ & $\begin{array}{l}153.00(95.50 \\
210.50)\end{array}$ & $<0.001$ & 0.716 & 0.005 & 0.006 \\
\hline $\begin{array}{c}\text { 总胆红素 }\left(\mathrm{mg} \mathrm{dL}^{-1} \text {, 中位数 }\right. \\
[\mathrm{IQR}])\end{array}$ & $\begin{array}{l}227.90(170.85 \\
306.75)\end{array}$ & $\begin{array}{c}302.90 \\
(155.38,499.95)\end{array}$ & $51.60(17.50,154.60)$ & $<0.001$ & 1.00 & 0.002 & $<0.001$ \\
\hline $\begin{array}{c}\gamma \text {-谷酰胺转酞酶 }\left(\mathrm{U} \mathrm{L}^{-1},\right. \\
\text { 中位数 }[\mathrm{IQR}])\end{array}$ & $\begin{array}{c}144.00(98.50 \\
224.75)\end{array}$ & $\begin{array}{l}180.00(105.50 \\
281.00)\end{array}$ & $92.00(44.00,187.00)$ & 0.026 & 0.566 & 0.042 & 0.021 \\
\hline 国际标准化比值 (中位数[IQR]) & $1.09(1.01,1.23)$ & $1.14(1.02,1.40)$ & $0.95(0.85,1.03)$ & $<0.001$ & 1.00 & 0.002 & $<0.001$ \\
\hline $\begin{array}{l}\text { 总胆固醇 }\left(\mathrm{mmol} \mathrm{L}^{-1},\right. \\
\text { 中位数 }[\mathrm{IQR}])\end{array}$ & $3.64(2.80,4.09)$ & $3.85(2.42,4.64)$ & $4.86(3.85,5.69)$ & $<0.001$ & 1.00 & 0.085 & $<0.001$ \\
\hline $\begin{array}{c}\text { 总甘油酯 }\left(\mathrm{mmol} \mathrm{L}^{-1},\right. \\
\text { 中位数 }[\mathrm{IQR}])\end{array}$ & $2.70(1.82,3.51)$ & $2.65(1.47,3.82)$ & $2.17(1.53,2.59)$ & 0.022 & 1.00 & 0.532 & 0.02 \\
\hline $\begin{array}{l}\text { 血清白蛋白 }\left(\mathrm{g} \mathrm{L}^{-1},\right. \\
\text { 中位数 }[\mathrm{IQR}])\end{array}$ & $34.00(31.25,37.75)$ & $32.00(24.00,37.50)$ & $37.00(30.50,41.00)$ & 0.08 & - & - & - \\
\hline 胆碱酯酶 $\left(\mathrm{U} \mathrm{L}^{-1}\right.$, 中位数 $\left.[\mathrm{IQR}]\right)$ & $\begin{array}{l}4751.00(3390.00 \\
5521.25)\end{array}$ & $\begin{array}{l}3718.50(2123.75 \\
6079.25)\end{array}$ & $\begin{array}{c}6089.00(4705.00 \\
7976.50)\end{array}$ & $<0.001$ & 1.00 & 0.008 & 0.002 \\
\hline
\end{tabular}

* a): 三组间的显著性差异分析; b): DILI组与ALD-DILI组显著性差异分析; c): ALD-DILI组与ALD组显著性差异分析; d): DILI组与ALD组 显著性差异分析. b), c), d): 用卡方检验或Fisher's精确检验分析组间分类变量差异, a用Bonferroni’s校正多项比较结果. DILI: 药物性肝损伤; ALD: 酒精性肝病; ALD-DILI, 酒精性肝病合并药物性肝损伤; IQR: 四分位距 $(25 \%$ \% $75 \%)$ 
A
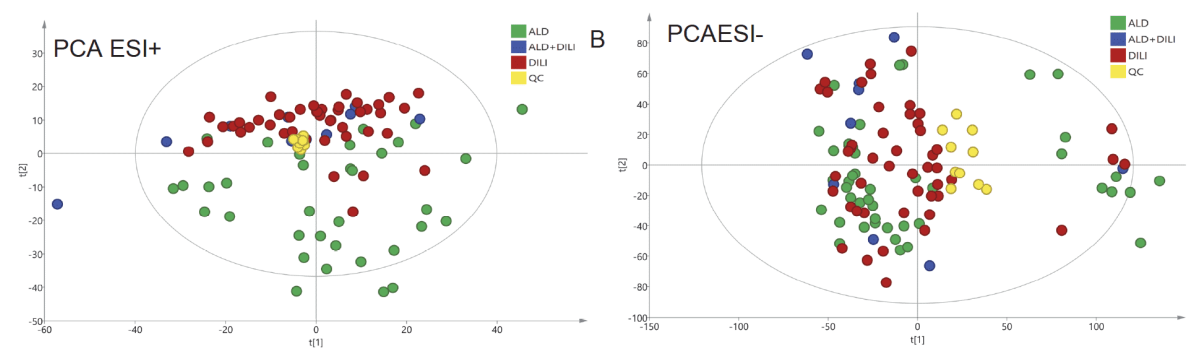

C
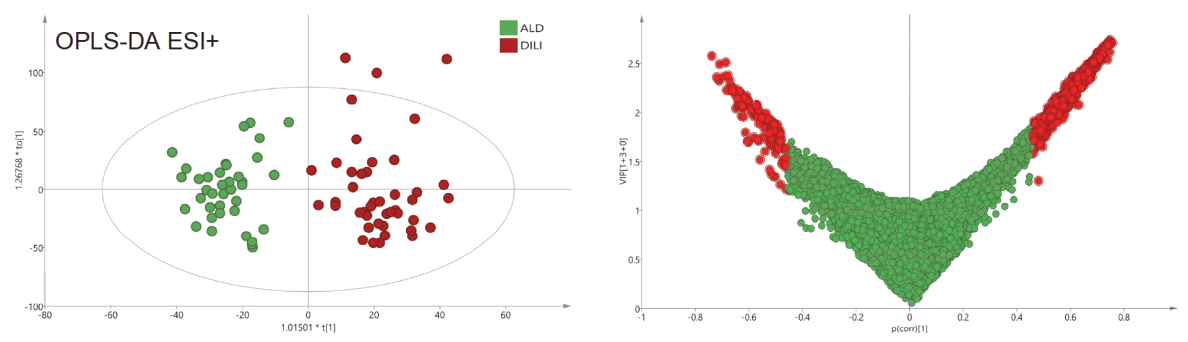

D
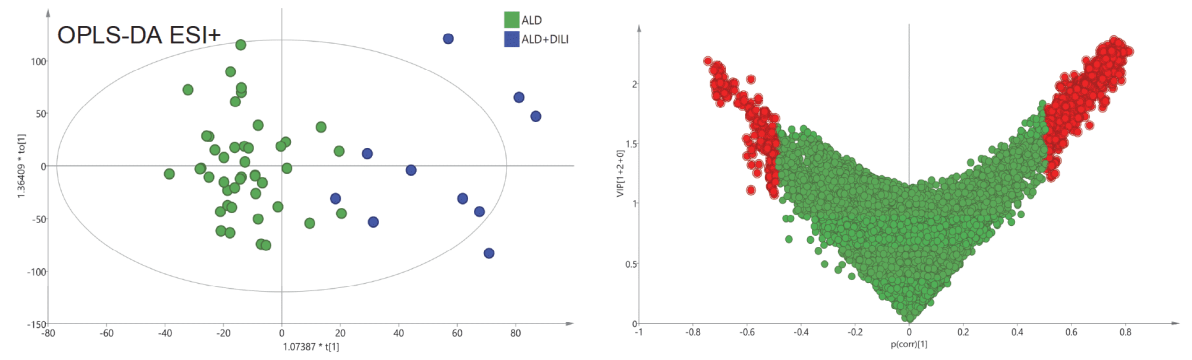

$\mathrm{E}$
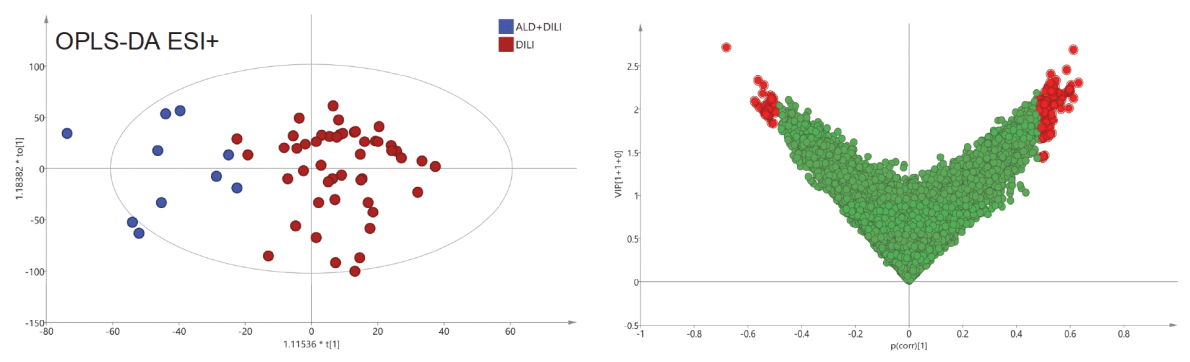

$\mathrm{F}$
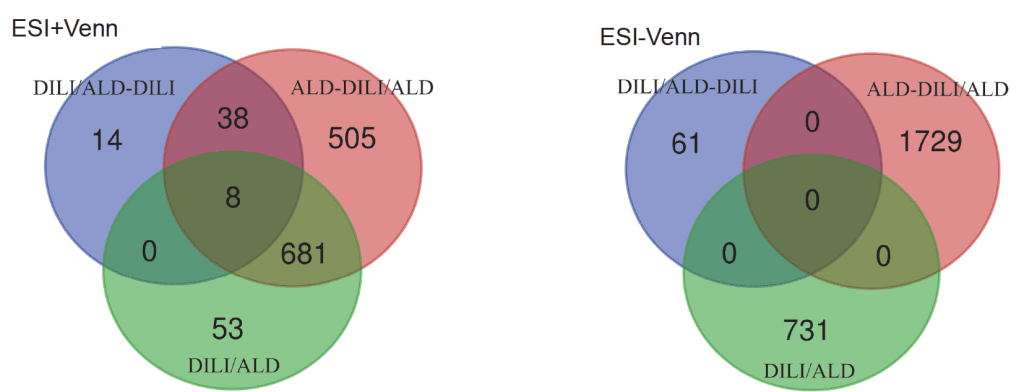

图 2 DILI, ALD, ALD-DILI三组的代谢组学分析. A, B: 正离子模式及负离子模式下PCA图; C E: 正离子模式下DILI与ALD, ALD-DILI与ALD, ALD-DILI与DILI的OPLS-DA图(左)和Scatter图(右); F: DILI与ALD-DILI, DILI与ALD, ALD-DILI与ALD组 间差异物的韦恩图

Figure 2 Metabolomic analysis among the DILI groups, ALD groups, DILI with pre-existing ALD groups. A, B: PCA score plots of different groups in ESI+ mode ESI- mode. C-E: OPLS-DA scores plot and S-plot of plasma metabolic profiling using the date from significantly changed metabolites among DILI group vs. ALD group, ALD-DILI group vs. ALD group, ALD-DILI group vs. DILI group. F: Venn diagram illustrates numbers of common and uniquely significantly changed metabolites in plasma of ALD-DILI group vs. DILI group, ALD-DILI group vs. ALD group, DILI group vs. ALD group 
寻找DILI，ALD-DILI，ALD两两之间的差异性化合物 (图2C $\sim \mathrm{E}$ ). 进一步采用MetaboAnalyst 3.0对三组进行 单变量分析, 包括ANOVA及倍数变化分析 $(P<0.05$ 且 $\mathrm{FC}>1.5$ 或 $<0.67)$, 分别筛选出三组间具有显著性差异 的化合物; 将上述篎选出的差异化合物通过韦恩图分 析(图2F), 可得到差异化合物不同对照组组间的分布 情况(图3D), 其中: DILI组与ALD-DILI组间有 60 个差 异化合物, ALD-DILI组与ALD组间有 1232 个差异化合 物，DILI组与ALD组间有 689 个差异化合物(在正离子 模式下).

\subsection{DILI, ALD-DILI, ALD间代谢特征谱分析}

通过软件OriginPro 8.5 将上述方法篮选到的差异 化合物以其相应的保留时间(retention time, RT)、质 量数(mass number, MZ)、倍数变化及 $P$ 值进行代谢特 征谱分析(图3A，B). 从正负离子模式下三组间的代谢 特征谱均可以看出, 从数量及倍数变化上DILI与ALDDILI组明显少于DILI与ALD组、ALD-DILI与ALD组. 进一步说明ALD合并DILI与ALD差异较大，与DILI表 现更类似，且存在一定的代谢差异.

\section{4 代谢组学生物标志物篮选及通路分析}

将正负离子篮选出的差异化合物在Human Metabolome Database和Mass Hunter PCDL Manager (MHPM)等生物学数据库进行比对笁查, 质量误差限 定为 $30 \mathrm{ppm}$, 最后鉴定出符合条件的差异化合物DILI/ ALD组119个、ALD-DILI/ALD组244个、DILI/ALDDILI组 28 个. 将这些符合条件的差异化合物通过Cytoscape version 3.6.1软件结合MetScape数据库进行总 的通路分析(图4A). 从图A中可以看出, 总的通路分析 图大致分成了两个子网：其中一部分以DILI，ALDDILI分别与ALD进行比较后的差异化合物为主，其影 响的代谢过程较为相似，主要包括氨基酸代谢、胆汁 酸代谢、脂类代谢等过程; 另一部分以DILI组与ALDDILI组比较的差异代谢物为主, 影响的代谢通路主要 是色氨酸代谢、甘油磷脂代谢、甘油酯代谢过程. 为 了进一步分析DILI与ALD-DILI间的机制差异，将 DILI/ALD-DILI组的鉴定到的 28 个差异化合物单独进 行通路分析(图4B). 从图4B中可以看出, DILI与ALDDILI的差异化合物主要集中在甘油磷脂、胆汁酸、叶 酸、半乳糖等代谢通路上, 涉及 12 个差异代谢物(表2).
针对部分差异代谢物进行质谱确证，结果见图4C, 色 氨酸、牛磺酸、乳酸和 3 -吲哚乙酸的 4 个化合物二级 碎片离子图谱与标准谱图基本一致.

\subsection{DILI与ALD-DILI差异代谢物热图及ROC曲 线分析}

对DILI与ALD-DILI组代谢通路中的候选生物标 志物(表2)进行热图分析，发现部分差异化合物能较好 地区分DILI和ALD-DILI(图5A). 结果提示其有一定的 能辅助诊断DILI与ALD-DILI的功能, 同时在某种程度 上也可能与DILI与ALD-DILI的机制差异有关. 将这些 差异化合物进行ROC曲线分析, 有 4 个差异化合物对于 两组区分效果较好 $(A U C>0.8)$, 分别是Taurolithocholate, Phosphatidylethanolamine, 3-Ketolactose, Phosphatidate(图5B). 为了进一步提高生物标志物临床区分诊 断ALD-DILI的适用性, 避免不同实验室仪器差异及样 本处理过程的操作误差，采用相对定量的方法(图5C), 将这 4 个差异化合物的峰面积做比值进行相对定量, 结果发现相较于单独的Phosphatidate和Taurolithocholate，应用这两者的峰面积比值区分DILI与ALD-DILI 效果更好, AUC为 0.918 ; 其次为Phosphatidate与 $3-\mathrm{Ke}-$ tolactose 的峰面积比值, AUC为0.886(图5D).

\section{3 讨论}

随着社会经济的发展，酒精性肝病(ALD)合并药 物性肝损伤(DILI) 是现今大多数国家关注的一个热点

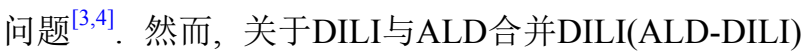
之间的机制差异研究有待深入. 本课题组前期研究发 现，酒精性肝病是慢性基础肝病合并DILI中的主要类 型，而ALD-DILI在临床生化指标表型上更接近于单纯 的DILI. 本研究在此基础上, 采用代谢组学的方法初步 探讨分析DILI, ALD-DILI, ALD间在生物组学水平上 的差异. 首先，从图2多元统计分析的结果可以看出 ALD-DILI与DILI重叠部分更多，说明这两者更相似， 而与ALD相差较大; 图3三组间的代谢谱中，气泡代表 的差异代谢物以 $\mathrm{B}, \mathrm{E}$ 中最多, A, D中最少, 进一步说明 ALD-DILI与DILI更相似. 图2与3均从代谢组表征上表 明了ALD-DILI更类似于DILI的客观真实性. 本文的研 究结果支持了前期课题组临床观察的结论，即在ALD 合并DILI的患者与DILI更相似，建议对ALD-DILI患者 
A

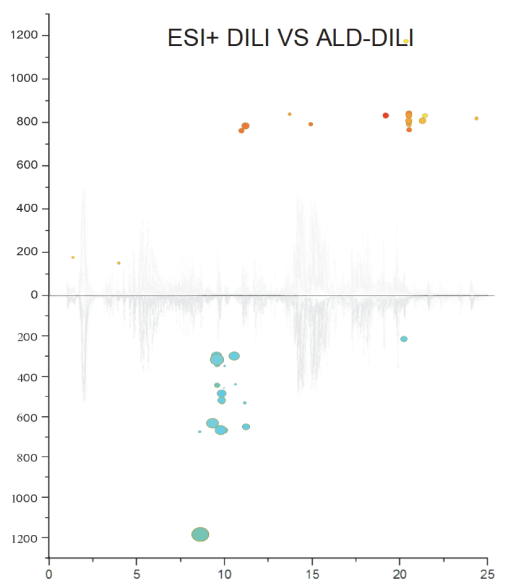

B

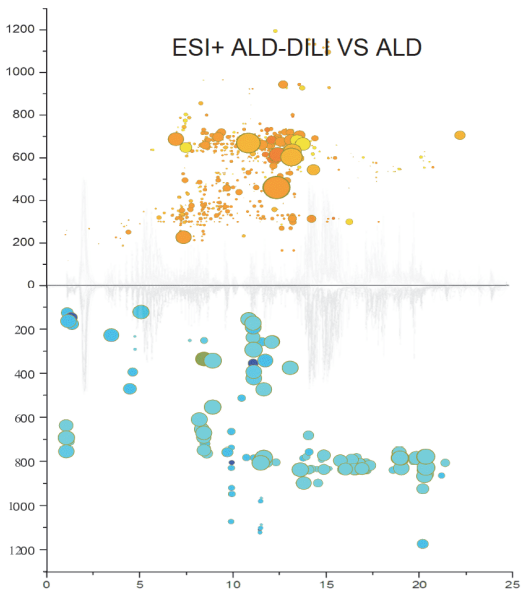

C

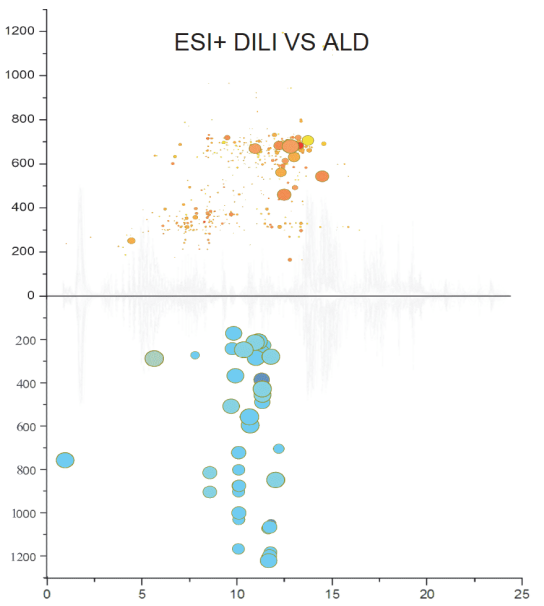

D

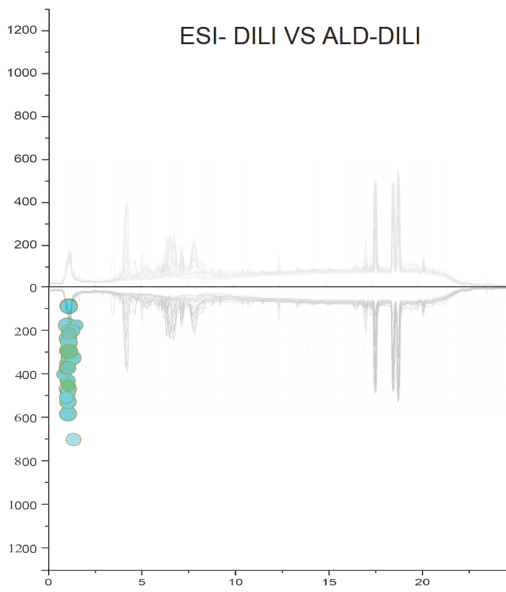

E 1200

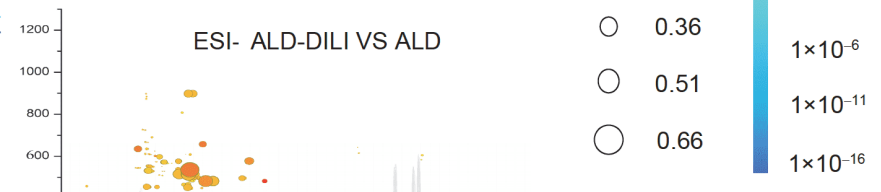

图 3 DILI, ALD, ALD-DILI三组间的代谢特征谱分析. 气泡代表DILI, ALD, ALD-DILI间差异代谢物, 其中横坐标是保留时 间 $(\mathrm{RT})$, 纵坐标是分子量 $(\mathrm{MZ})$, 气泡大小代表倍数变化 $(\mathrm{FC})$, 气泡颜色由深到浅代表 $P$ 值由小到大 $(P<0.05, \mathrm{FC}>1.5$ 或 $<0.67)$. 中 间0轴上下的橙色系代表FC $>1.5$, 蓝色系代表FC $<0.67$

Figure 3 Metabolomic profile analysis between DILI group, ALD group and ALD-DILI group. Cloud plot showing metabolite features (represented by bubbles) whose lever vary significantly between DILI, ALD and ALD-DILI serum. The abscissa is RT and the ordinate is MZ. The fold change is used as radius scale of each bubble. Darker color of the bubble indicates lower $P$-value $(P<0.05, \mathrm{FC}>1.5$ or $<0.67)$. The orange line above the center 0 axis represents $\mathrm{FC}>1.5$, and the blue line below the center 0 axis represents $\mathrm{FC}<0.67$ 

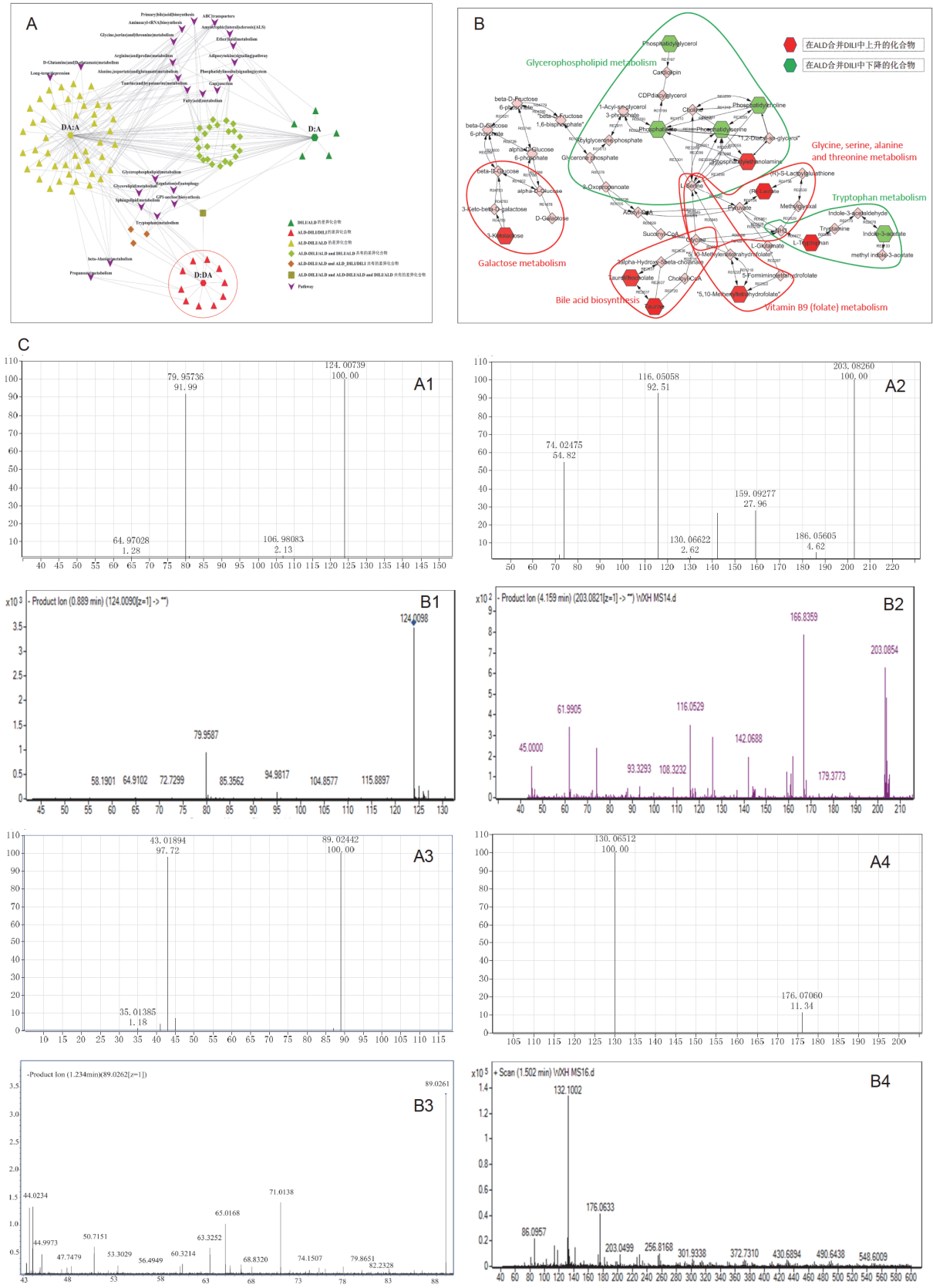

图 4 通路分析及标志物鉴定. A: DILI, ALD-DILI与ALD三者间差异化合物所在的总代谢通路图. DA:A表示ALD-DILI vs. ALD, D:A表示DILI vs. ALD, D:DA表示DILI vs. ALD-DILI; B: ALD-DILI与DILI差异化合物代谢通路分析. 六边形表示ALDDILI与DILI患者血清中鉴定到的差异化合物，菱形表示差异化合物所在通路中关联的化合物; 绿色六边形与绿色圆圈表示该 差异化合物及其所在通路在ALD-DILI呈下调的趋势，红色六边形与红色圆圈表示该差异化合物及其所在通路在ALD-DILI呈 上调的趋势. C: 患者血清中牛磺酸、色氨酸、乳酸和3-吲哚乙酸的标准质谱(A1 A4)及患者血清中四种化合物的MS/MS图谱 (B1 B4)

Figure 4 Pathway analysis and marker identification. A: Metabolic pathway analysis of the differential compounds between DILI, ALD-DILI and ALD. DA:A means ALD-DILI vs. ALD, D:A means DILI vs. ALD, D:DA means DILI vs. ALD-DILI. B: Analysis of metabolic pathways of differential compounds between ALD-DILI and DILI. Hexagons represent the differential compounds identified in the serum of ALD-DILI and DILI patients, and the diamonds indicate the compounds associated with the pathway; green hexagons and green circles indicate the tendency of the differential compound and its pathway to be down-regulated in ALD-DILI, and the red hexagons and red circles indicate the tendency of the differential compound and its pathway to be up-regulated in ALD-DILI. C: Standard mass spectrum (A1-A4) of taurine, tryptophan, $(R)$-lactate and indole-3-acetate in patient serum and MS/MS spectra of four compounds in patient serum (B1-B4) 
表 2 DILI与ALD-DILI 潜在标志物的鉴定结果及其代谢通路

Table 2 Identification biomarkers for DILI vs. ALD-DILI and their pathways

\begin{tabular}{|c|c|c|c|c|c|c|c|}
\hline \multirow{2}{*}{ 序号 } & \multirow{2}{*}{ 分子式 } & \multirow{2}{*}{ 分子量(ppm) } & \multirow{2}{*}{ 保留时间(min) } & \multirow{2}{*}{ 代谢物 } & \multirow{2}{*}{ 代谢通路 } & \multicolumn{2}{|c|}{ DILI/ALD-DILI } \\
\hline & & & & & & $\mathrm{FC}$ & $P$ \\
\hline \multicolumn{8}{|l|}{ ESI+ } \\
\hline 1 & $\mathrm{C}_{46} \mathrm{H}_{81} \mathrm{NO}_{8} \mathrm{P}$ & 806.5663 & 21.23 & $\mathrm{PC}(16: 1(9 \mathrm{Z}) / 14: 1(9 \mathrm{Z}))$ & 甘油磷脂代谢 & 3.43 & 0.0465 \\
\hline 2 & $\mathrm{C}_{42} \mathrm{H}_{80} \mathrm{NO}_{10} \mathrm{P}$ & 790.5706 & 20.47 & $\operatorname{PS}(18: 1(9 \mathrm{Z}) / 18: 0)[\mathrm{U}]$ & 甘油磷脂代谢 & 2.99 & 0.0182 \\
\hline 3 & $\mathrm{C}_{43} \mathrm{H}_{83} \mathrm{O}_{10} \mathrm{P}$ & 791.5716 & 20.47 & $\mathrm{PG}(22: 1(11 Z) / 15: 0)$ & 甘油磷脂代谢 & 2.69 & 0.0235 \\
\hline 4 & $\mathrm{C}_{46} \mathrm{H}_{79} \mathrm{O}_{8} \mathrm{P}$ & 791.5669 & 14.89 & $\begin{array}{c}\mathrm{PA}(21: 0 / 22: 6 \\
(4 \mathrm{Z}, 7 \mathrm{Z}, 10 \mathrm{Z}, 13 \mathrm{Z}, 16 \mathrm{Z}, 19 \mathrm{Z}))\end{array}$ & 甘油磷脂代谢 & 2.51 & 0.0010 \\
\hline 5 & $\mathrm{C}_{26} \mathrm{H}_{45} \mathrm{NO}_{5} \mathrm{~S}$ & 484.2965 & 9.7 & Taurolithocholate & 胆汁酸代谢 & 0.35 & 0.0004 \\
\hline 6 & $\mathrm{C}_{12} \mathrm{H}_{20} \mathrm{O}_{11}$ & 341.1137 & 9.44 & 3-Ketolactose & 半乳糖代谢 & 0.23 & 0.0002 \\
\hline 7 & $\mathrm{C}_{20} \mathrm{H}_{22} \mathrm{~N}_{7} \mathrm{O}_{6}$ & 457.1668 & 9.82 & 5,10-Methenyltetrahydrofolate & 叶酸代谢 & 0.07 & 0.0026 \\
\hline 8 & $\mathrm{C}_{10} \mathrm{H}_{9} \mathrm{NO}_{2}$ & 176.0655 & 1.36 & Indole-3-acetate & 色氨酸代谢 & 1.56 & 0.0240 \\
\hline \multicolumn{8}{|l|}{ ESI- } \\
\hline 9 & $\mathrm{C}_{38} \mathrm{H}_{74} \mathrm{NO}_{8} \mathrm{P}$ & 702.5073 & 1.35 & $\operatorname{PE}(18: 1(11 Z) / 15: 0)$ & 甘油磷脂代谢 & 0.56 & 0.0004 \\
\hline 10 & $\mathrm{C}_{3} \mathrm{H}_{6} \mathrm{O}_{3}$ & 89.0245 & 1.09 & $(R)$-Lactate & $\begin{array}{c}\text { 甘氨酸丝氨酸丙氨 } \\
\text { 酸苏氨酸代谢 }\end{array}$ & 0.67 & 0.0037 \\
\hline 11 & $\mathrm{C}_{2} \mathrm{H}_{7} \mathrm{NO}_{3} \mathrm{~S}$ & 124.0090 & 1.11 & Taurine & 胆汁酸代谢 & 0.56 & 0.0149 \\
\hline 12 & $\mathrm{C}_{11} \mathrm{H}_{12} \mathrm{~N}_{2} \mathrm{O}_{2}$ & 203.0821 & 2.45 & $L$-Tryptophan & 色氨酸代谢 & 0.56 & 0.0143 \\
\hline
\end{tabular}

可以主要按DILI的方法进行管理与治疗, 降低ALDDILI患者的死亡率.

其次，对DILI，ALD-DILI之间的机制差异进行了 初步探讨. 根据图4A对三种疾病之间差异代谢物的分 析结果，ALD-DILI与ALD之间的差异代谢物(黄色标 识)最多, 其中包含了大部分DILI与ALD之间的差异代 谢物(绿色标识)，表明ALD-DILI既具有类似DILI的代 谢特征，又与DILI之间存在一定差异，但是这种差异 多数在ALD-DILI与DILI组间并不显著，而ALD-DILI 与DILI的代谢差异具有自身的特点(红色标识). 进一 步, 针对ALD-DILI与DILI的差异代谢物进行通路分析 (图4B)，共筛选12个潜在生物标志物，包括磷脂酰甘 油、磷脂酰乙醇胺、磷脂酰胆碱、磷脂酰丝氨酸、磷 脂酸、牛磺石胆酸、3-酮基乳糖、 $(R)$-乳酸、 5,10 -甲 川四氢叶酸、吲哚乙酸(表2)，主要涉及甘油磷脂、胆 汁酸、氨基酸、半乳糖、色氨酸、叶酸等代谢通路. 其中, 相对于DILI而言, ALD-DILI患者PG, PC, PA和 $\mathrm{PS}$ 等磷脂均降低，而PE则显著升高，这与长期的、慢 性的酒精性肝病中, 磷脂类代谢表现极为相似 ${ }^{[16,17]}$. 同 时，ALD-DILI患者体内牛磺石胆酸、 $(R)$-乳酸、 $L$-色
氨酸均表现出一定程度的升高. 有研究显示, 乙醇可 促进体内半乳糖代谢 ${ }^{[18]}$ 和肝糖酵解, 可导致D-半乳糖 代谢产物3-Ketolactose含量升高; 牛磺石胆酸(taurolithocholic acid, TLCA)是一种具有潜在肝毒性的次生 胆汁盐 ${ }^{[19]}$, 可能通过直接作用于胆管膜而引起胆汁淤 积，从而损害胆管收缩和胆管分泌 ${ }^{[20,21]}$ ，而血清牛磺 石胆酸升高亦常见于肝硬化等终末期肝病 ${ }^{[22]}$; 此外, 急性酒精中毒可导致大量乳酸堆积 ${ }^{[23]}$, 而乳酸升高与 肝衰竭的发生具有一定相关性 ${ }^{[24]}$, 而色氨酸升高亦可 一定程度导致肝性脑病的发生 ${ }^{[25]}$. 上述结果均表明, ALD-DILI患者具有一定ALD的代谢特征, 且部分代谢 物的升高可能与许多严重的并发症相关, 这可能是本 课题组前期临床观察中ALD-DILI患者治愈率更低的 因素之一 ${ }^{[8]}$.

由此可见，代谢组学方法篮选发现ALD-DILI与 DILI间的差异代谢物与其发生机制的差异密切相关, 从中进一步篮选可能具有辅助诊断能力的生物标志 物, 对于早期鉴别诊断ALD-DILI与DILI患者并指导 临床用药具有较好的前景和现实意义, 特别是那些具 有长期饮酒史却没有发生ALD的DILI患者. 为此, 我 
A

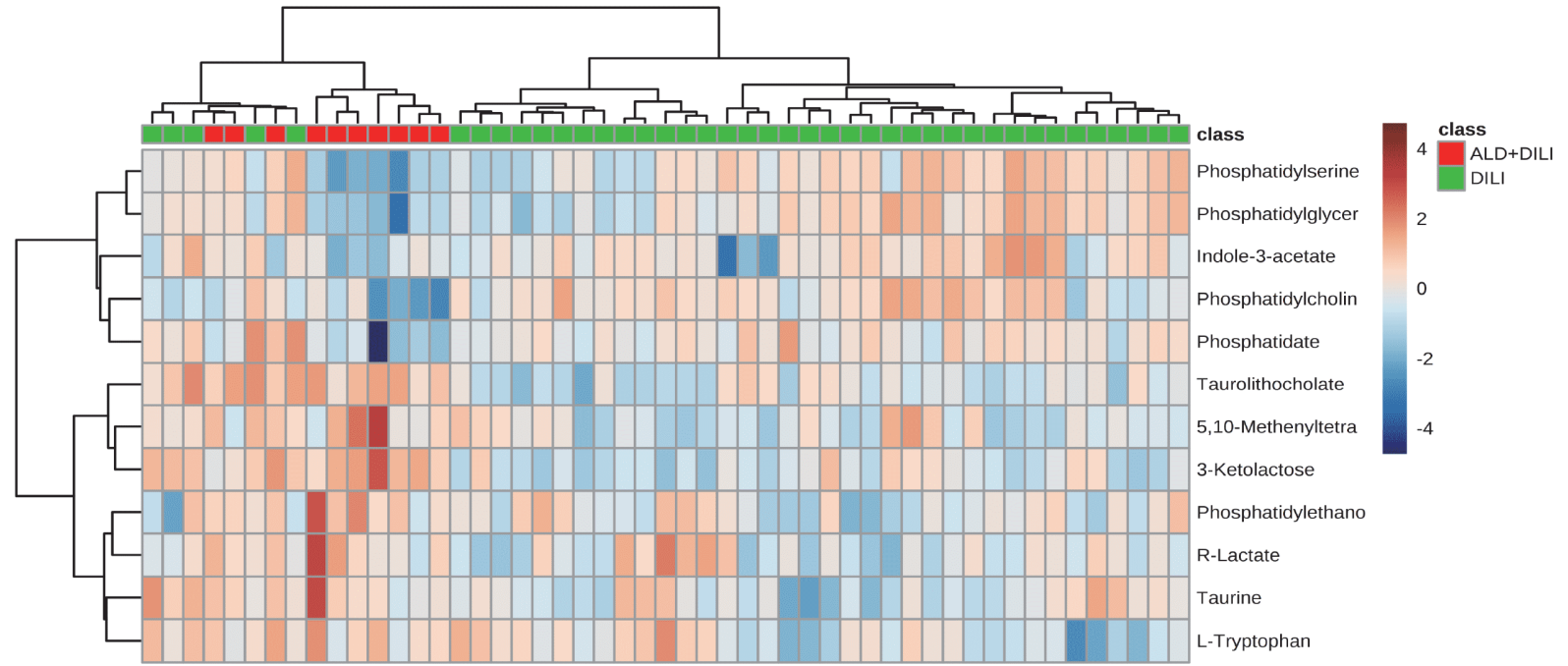

B

Taurolithocholate

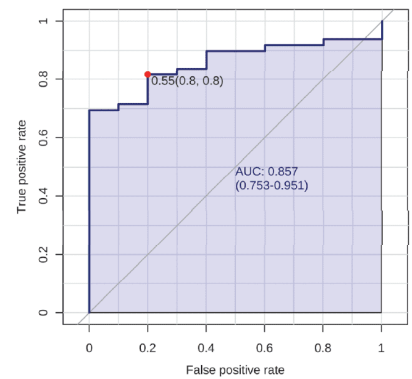

C
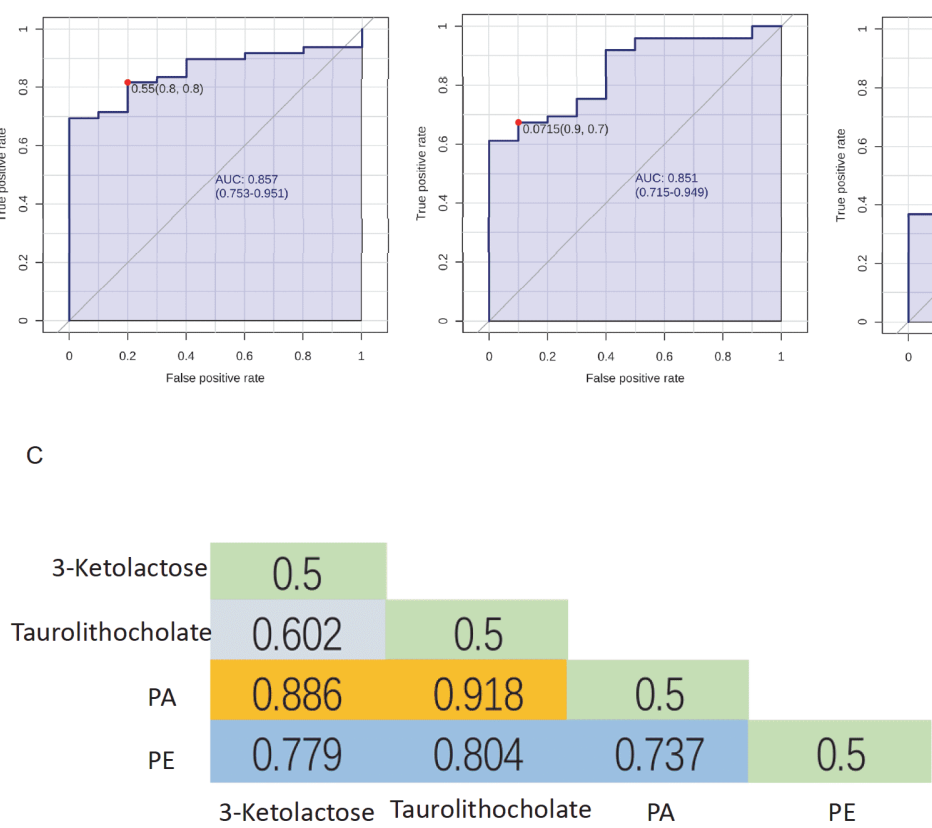

$\operatorname{PE}(18: 1(11 Z) / 15: 0$
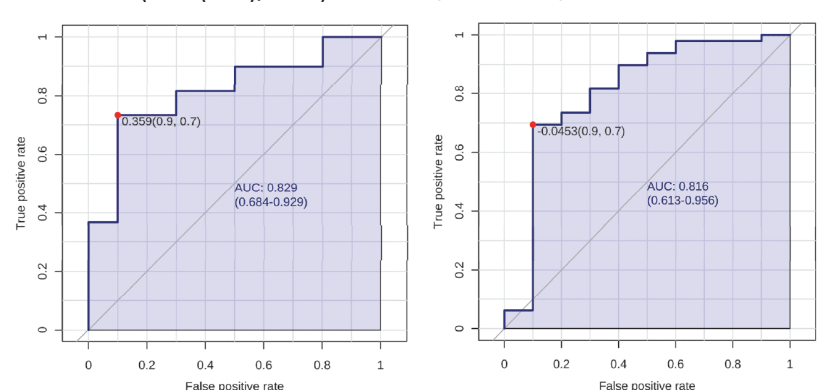

False positive rate

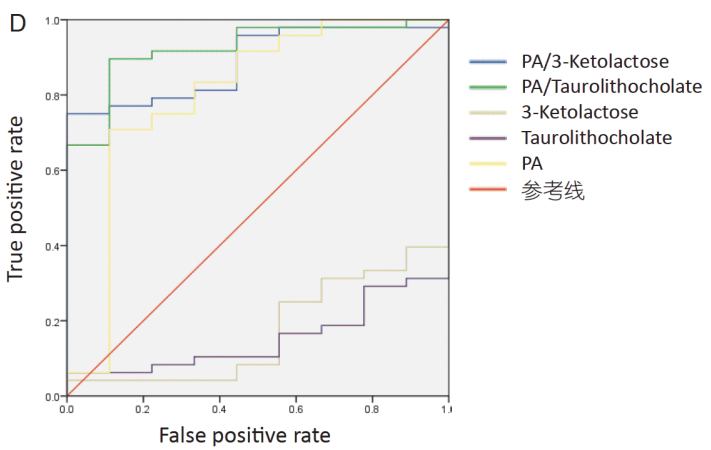

图 5 DILI与ALD-DILI差异代谢物热图及ROC曲线分析. A: DILI与ALD-DILI差异代谢物的热图聚类分析, 颜色代表不同样 本间的区分程度；B：DILI与ALD-DILI间潜在生物标志物的ROC曲线分析 $(A U C>0.8) ; C$ : 潜在生物标志物的峰面积比值; D: Phosphatidate, 3-Ketolactose, Taurolithocholate单独及进行峰面积比值后的ROC曲线对比分析

Figure 5 Analysis of related metabolites in Pathway. A: Clustered heat map analysis of differential metabolites in DILI and ALD-DILI, the colors represent the contents in each of differentially expressed. B: The ROC curve of potential metabolites biomarkers associated with DILI and ALD-DILI $(A U C>0.8)$. C: AUC of the metabolites peak area ratio. D: AUC of ROC curves of Phosphatidate, 3-Ketolactose, Taurolithocholate alone and peak area ratio

们通过ROC对代谢物的区分效果进行预测，发现有 4 个差异代谢物的区分效果较好(AUC均大于 0.8 ), 分别 是Phosphatidate, Phosphatidylethanolamine, Taurolithocholate, 3-Ketolactose)(图5A，B)。然而，在临床
上应用生物标志物区分诊断疾病时如采用绝对定量, 需要高度纯化的代谢物作为对照品, 获得难度和成本 较高; 此外生物标志物绝对定量的实验结果往往受仪 器和操作等因素影响, 对不同实验室检测结果的重现 
性要求较高. 因此, 本研究考虑可采用两个代谢物的 峰面积比值进行相对定量, 以期提高候选生物标志物 的可应用性和稳定性. 通过对所发现的 4 个代谢物进 行两两组合构建峰面积比值后进行ROC分析(图 5C, D), 结果显示Phosphatidate分别与Taurolithocholate, 3-Ketolactose的比值高达 0.918 和 0.886 , 区分ALDDILI与DILI能力比单独应用代谢物的效果更好. 同时
还可以更好地克服因仪器和操作不稳定等实验因素 造成的系统误差，具有更稳健的临床应用价值. 因此 可以将Phosphatidate分别与 Taurolithocholate, 3-Ketolactose的两个脂质相对定量作为临床上辅助诊断 ALD-DILI与DILI的生物标志物组合. 后续将在更大 样本临床队列中对所发现的候选生物标志物进行 验证.

\section{参考文献}

1 Tang Y, Xiang X, Wang X, et al. Alcohol and alcohol-related harm in China: Policy changes needed. Bull World Health Organ, 2013, 91: 270276

2 Li Y M. Epidemiological characteristics of alcoholic liver disease (in Chinese). Pract Liver Dis J, 2012, 15: 180-182 [厉有名. 酒精性肝病的流 行病学特点. 实用肝脏病杂志, 2012, 15: 180-182]

3 Mathurin P, Bataller R. Trends in the management and burden of alcoholic liver disease. J Hepatol, 2015, 62: S38-S46

4 Gao B, Bataller R. Alcoholic liver disease: Pathogenesis and new therapeutic targets. Gastroenterology, 2011, 141: 1572-1585

5 Rehm J, Mathers C, Popova S, et al. Global burden of disease and injury and economic cost attributable to alcohol use and alcohol-use disorders. Lancet, 2009, 373: 2223-2233

6 Zimmerman H J. Hepatotoxicity: The adverse effects of drugs and other chemicals on the liver. Gastroenterology, 1999, 118: 984-985

7 O’Dell J R, Zetterman R K, Burnett D A. Centrilobular hepatic fibrosis following acetaminophen-induced hepatic necrosis in an alcoholic. JAMA, 1986, 255: 2636

8 Jing J, Wang R L, Zhao X Y, et al. Association between the concurrence of pre-existing chronic liver disease and worse prognosis in patients with an herb-Polygonum multiflorum thunb. induced liver injury: A case-control study from a specialised liver disease center in China. BMJ Open, 2019, 9: e023567

9 Fontana R J, Hayashi P H, Gu J, et al. Idiosyncratic drug-induced liver injury is associated with substantial morbidity and mortality within 6 months from onset. Gastroenterology, 2014, 147: 96-108.e4

10 Chinese Medical Association Liver Disease Branch, Pharmacological Hepatology Group. Guidelines for the diagnosis and treatment of druginduced liver injury (in Chinese). Chin J Hepatol, 2015, 23: 810-820 [中华医学会肝病学分会药物性肝病学组. 药物性肝损伤诊治指南. 中华 肝脏病杂志, 2015, 23: 810-820]

11 Xiao X, Tang J, Mao Y, et al. Guidance for the clinical evaluation of traditional Chinese medicine-induced liver injury Issued by China Food and Drug Administration. Acta Pharmaceut Sin B, 2018, doi: 10.1016/j.apsb.2018.12.003

12 Xiao X H, Tang J Y, Mao Y M, et al. Technical guidelines for clinical evaluation of traditional Chinese medicine-induced liver injury (in Chinese). J Pharm, 2018, 53: 1931-1942 [肖小河, 唐健元, 茅益民, 等. 中药药源性肝损伤临床评价技术指导原则. 药学学报, 2018, 53: 19311942]

13 Xiao X H, Li X H, Zhu Y, et al. Guideline for the diagnosis and treatment of herb-induced liver injury (in Chinese). Chin J Trad Chin Med, 2016, 41: 1165 [肖小河, 李秀惠, 朱云, 等. 中草药相关肝损伤临床诊疗指南. 中国中药杂志, 2016, 41: 1165

14 Wang J B, Zhu Y, Bai Z F, et al. Guidelines for the diagnosis and management of herb-induced liver injury. Chin J Integr Med, 2018, 24: 696-706

15 Chinese Medical Association Liver Diseases Branch Fatty Liver and Alcoholic Liver Disease Group. Guideline for the diagnosis and treatment of alcoholic liver disease (in Chinese). Chin J Hepatol, 2010, 2: 49-53 [中华医学会肝病学分会脂肪肝和酒精性肝病学组. 酒精性肝病诊疗指南. 中国肝脏病杂志, 2010, 2: 49-53]

16 Lieber C S, Casini A, Decarli L M, et al. S-adenosyl-L-methionine attenuates alcohol-induced liver injury in the Baboon. Hepatology, 2010, 11: $165-172$

17 Li H, Xu W, Jiang L, et al. Lipidomic signature of serum from the rats exposed to alcohol for one year. Toxicol Lett, 2018, 294: 166-176

18 Guo C, Chen L, Huang J, et al. Aldose reductase inhibitor protects mice from alcoholic steatosis by repressing saturated fatty acid biosynthesis. Chemico-Biol Interact, 2018, 287: 41-48 
19 Bove K E, Heubi J E, Balistreri W F, et al. Bile acid synthetic defects and liver disease: A comprehensive review BAS defects and liver disease. Pediatr Dev Pathol, 2004, 7: 315-334

20 Javitt N B. Cholestasis in rats induced by taurolithocholate. Nature, 1966, 210: 1262-1263

21 Watanabe N, Kagawa T, Kojima S, et al. Taurolithocholate impairs bile canalicular motility and canalicular bile secretion in isolated rat hepatocyte couplets. World J Gastroenterol, 2006, 12: 5320-5325

22 Li G X, Li P, Gao B. Determination of serum-bound bile acids in patients with bile acid and cirrhosis by reversed-phase high performance liquid chromatography (in Chinese). J Sichuan Univ (Med Ed), 2004, 35: 117-119 [李贵星, 李萍, 高兵. 反相高效液相色谱法测定结合胆汁酸及肝 硬化患者血清结合型胆汁酸分析. 四川大学学报(医学版), 2004, 35: 117-119]

$23 \mathrm{Hu} \mathrm{H} \mathrm{Y,} \mathrm{Zhou} \mathrm{L} \mathrm{Y.} \mathrm{Blood} \mathrm{gas} \mathrm{analysis} \mathrm{and} \mathrm{hemorheology} \mathrm{changes} \mathrm{and} \mathrm{significance} \mathrm{in} \mathrm{patients} \mathrm{with} \mathrm{acute} \mathrm{severe} \mathrm{alcoholism} \mathrm{(in} \mathrm{Chinese).}$ Guangdong Med, 2014, 35: 1535-1536 [胡鸿宇, 周凌燕. 急性重度酒精中毒患者血气分析及血液流变学的变化及意义. 广东医学, 2014, 35: 1535-1536]

$24 \mathrm{Xu} \mathrm{Z} \mathrm{Y,} \mathrm{Rao} \mathrm{Y,} \mathrm{Cheng} \mathrm{Y,} \mathrm{et} \mathrm{al.} \mathrm{Changes} \mathrm{of} \mathrm{plasma} \mathrm{D-lactic} \mathrm{acid,} \mathrm{diamine} \mathrm{oxidase} \mathrm{and} \mathrm{endotoxin} \mathrm{in} \mathrm{rats} \mathrm{with} \mathrm{acute} \mathrm{liver} \mathrm{failure} \mathrm{and} \mathrm{its} \mathrm{significance}$ (in Chinese). J Gastroenterol Hepatol, 2011, 20: 736-738 [徐智媛, 尧颖, 程宇, 等. 急性肝衰竭大鼠血浆D-乳酸、二胺氧化酶和内毒素的变 化及其意义. 胃肠病学和肝病学杂志, 2011, 20: 736-738]

25 Xu J Q, Li Q P. The role of tryptophan in the pathogenesis of hepatic encephalopathy (in Chinese). World Chin J Digestion, 1998, 6: 268-269 [徐 军全, 李秋萍. 色氨酸在肝性脑病发病机制中的作用. 世界华人消化杂志, 1998, 6: 268-269]

\title{
Metabolomic profiling and biomarkers of drug-induced liver injury with pre-existing alcoholic liver disease
}

\author{
WANG XiaoHui ${ }^{1,2}$, JING Jing ${ }^{2}$, NIU Ming ${ }^{2}$, WEI LuGe ${ }^{2}$, LIU XiaoYi ${ }^{2}$, TU Can ${ }^{2}$, BAI ZhaoFang ${ }^{2}$, \\ ZHOU YuanYuan ${ }^{2}$, ZHANG Le ${ }^{2}$, ZHU JingXiao ${ }^{2}$, XIAO XiaoHe ${ }^{4}$, ZOU ZhengSheng ${ }^{3}$ \\ \& WANG JiaBo ${ }^{2}$ \\ 1 College of Pharmacy, Hunan University of Traditional Chinese Medicine, Changsha 410200, China; \\ 2 China Military Institute of Chinese Medicine, The Fifth Medical Center, General Hospital of PLA, Beijing 100039, China; \\ 3 Center for Non-infectious Liver Diseases Diagnosis and Treatment, The Fifth Medical Center, General Hospital of PLA, Beijing 100039, China; \\ 4 Center for Integrative Medicine and Treatment, The Fifth Medical Center, General Hospital of PLA, Beijing 100039, China
}

With the development of social economy, the problem of drug-induced liver injury (DILI) with pre-existing alcoholic liver disease (ALD) is becoming serious. However, it is often difficult to define the dominant role of ALD combined with DILI (ALD-DILI), and the mechanism of damage pathway is not completely clear. In this paper, metabolomics characterization of samples showed that ALD-DILI was similar to DILI (60 different compounds) rather than ALD (1,232 different compounds) as a whole at the metabolome level. Through metabolite identification and metabolic pathway analysis, it was found that compared with DILI, functional injuries such as bile acid, amino acid and lipid synthesis and decomposition were more serious in ALD-DILI, which is consistent with the clinical results observed by our research group. Furthermore, 4 candidate biomarkers (Phosphatidate, Phosphatidylethanolamine, Taurolithocholate, 3-ketolactose) that can be used to distinguish DILI from ALD-DILI are further screened, and the area under the ROC curve is greater than 0.8. In addition, the ratio of Phosphatidate to 3-ketolactose and taurolithocholate has a better diagnosis effect, and the area under the ROC curve is 0.918 and 0.886 , respectively. The ratio method is conducive to reducing the systematic errors of sample processing and detection instruments, and can better assist in the early clinical differential diagnosis of ALD-DILI as well as guide clinical rational drug use.

drug-induced liver injury, alcoholic liver disease, metabolomics, biomarker, diagnosis

doi: $10.1360 / \mathrm{N} 052018-00291$ 